Supporting Information

\title{
Selective Extraction of Heavy and Light Lanthanides from Aqueous Solution by Advanced Magnetic Nanosorbents
}

\author{
Huijin Zhang ${ }^{1}$, Rocklan G. McDowell ${ }^{2}$, Leigh R. Martin², and You Qiang ${ }^{1,3 *}$ \\ ${ }^{1}$ Environmental Science Program, University of Idaho, Moscow, ID 83844 \\ ${ }^{2}$ Aqueous Separations and Radiochemistry Department, Idaho National Laboratory, Idaho Falls, \\ ID 83415 \\ ${ }^{3}$ Department of Physics, University of Idaho, Moscow, ID 83844
}

9 Pages, 8 Figures, 4 Tables

Submitted to ACS Applied Materials and Interfaces

* Corresponding author phone: (208)885-7558; fax (208)885-4055; e-mail: youqiang@ uidaho.edu. 


\section{Characterization of dMNP-DTPA nanosorbents:}

Vibrating Sample Magnetometer (VSM): Magentic properties were characterized using a DMS 1660 VSM where each pre-weighted sample is placed inside an external magnetic field to get magnetized at room temperature. A hysteresis loop was acquired by applying a scanning magnetic field of 13500 Oe to -13500 Oe.

Elemental analysis: Dry samples were sent to Midwest Microlabs, LLC (Indianapolis, IN) for determination of carbon $(\mathrm{C})$, hydrogen $(\mathrm{H})$, and nitrogen $(\mathrm{N})$ content. In this CHN analysis, a sample is burned in an excess of oxygen at $990^{\circ} \mathrm{C}$, and the combustion products (carbon dioxide, water, and nitric oxide) are collected by various traps. The masses of these combustion products can be used to calculate the $\mathrm{C}, \mathrm{H}, \mathrm{N}$ composition of the sample. The results of the elemental analysis were used to determine the loading density of DTPA onto the surface of dMNPs.

Fourier Transform Infrared Spectroscopy (FTIR): IR spectra of vacuum dry samples were obtained and analyzed on a Thermo-Nicolet Avatar 370 FTIR spectrometer (SmartPerformer, ZnSe crystal) operating in attenuated total reflectance (ATR) mode. A background scan of the ZnSe crystal was performed prior to measuring each sample. Each spectrum was taken as an average of 64 sample scans at a resolution of $4 \mathrm{~cm}^{-1}$. Moderate pressure was applied to the samples to ensure the good contact between samples and $\mathrm{ZnSe}$ crystal. The resultant spectra were baseline corrected. Special interest was focused on the signals assigned to the carboxylate group as well as the primary and secondary amine groups.

Transmission Electron Microscopy (TEM): Particle size and chemical composition was confirmed using TEM (Tecnai TF30-FEG STwin). The samples were prepared by drying the solvent of the MNPs' dispersion onto a 200 mesh copper grid with a carbon coated Formvar film. 
Inductively coupled plasma mass spectrometry (ICP-MS):Lanthanide concentrations in aqueous solutions were characterized using an ELAN 6000 ICP-MS (Perkin Elmer) before and after contact with the magnetic nanosorbents following the procedure described in the ref. ${ }^{1}$. The concentration of lanthanides in the sorbent phase was determined by the material balance equation.

\section{Properties of dMNP-DTPA:}

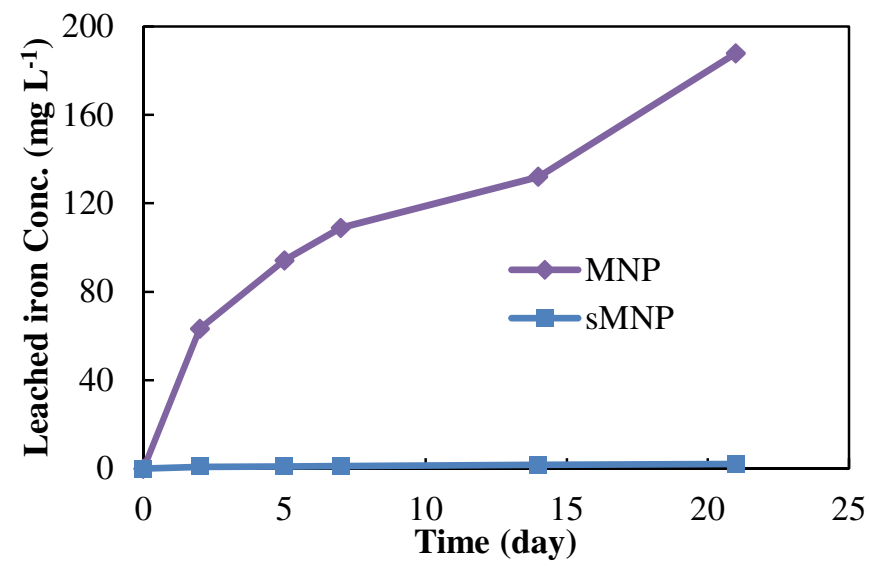

Figure S1. Time dependence of iron leaching from MNP and sMNP in $0.1 \mathrm{M} \mathrm{HNO}_{3}$.

Zeta potential data of dMNP and dMNP-DTPA as a function of $\mathrm{pH}$ are shown in Figure S2. The standard method used to transfer mobility data to zeta potential data assumes smooth hard particles. Therefore, the polymer surface structure of dMNP and dMNP-DTPA gives rise to a large uncertainty to the absolute zeta potential data. However, the sign of the zeta potential and the position of the point of zero charge (PZC) should be correct. In both cases, the data had positive values at low $\mathrm{pH}$, passed through a $\mathrm{PZC}$, and then turned to negative at high $\mathrm{pH}$. The zeta potential data suggested the surface charge of dMNP and dMNP-DTPA, which was highly related to their sorption power. 


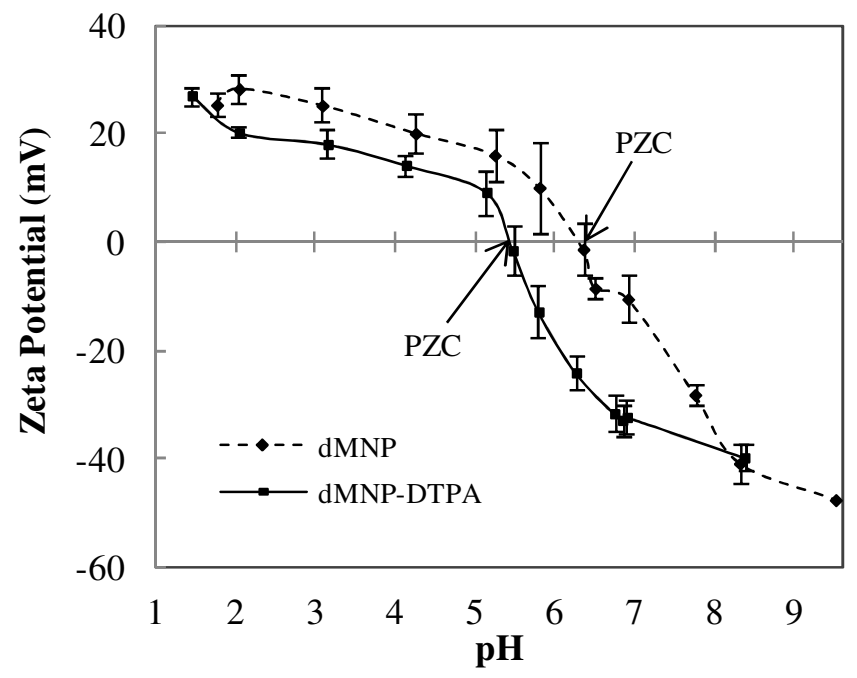

Figure S2. Zeta potential vs. $\mathrm{pH}$ for dMNP and dMNP-DTPA at a particle concentration of $30 \mu \mathrm{g} / \mathrm{mL}$.

\section{Ln(III) Sorption:}

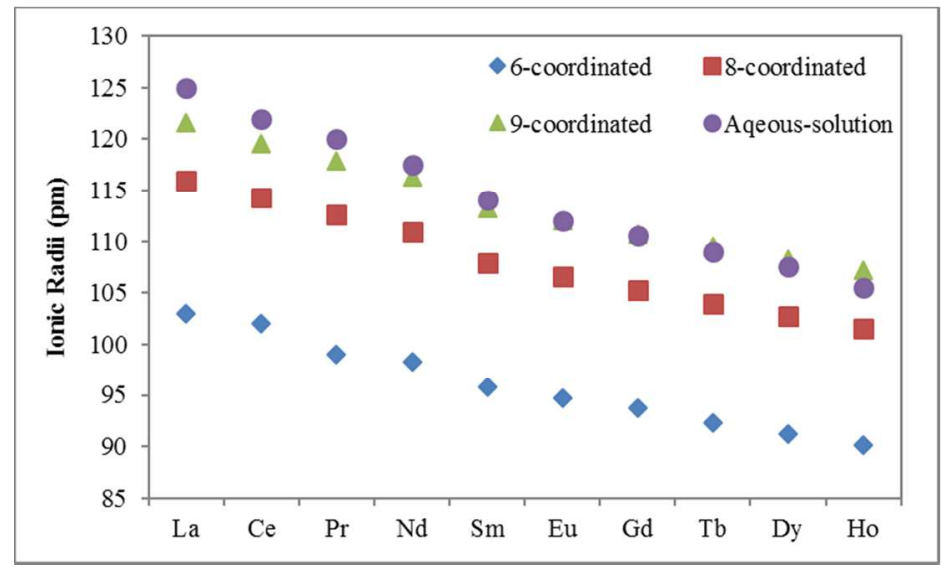

Figure S3. Crystal ionic radii (pm) of $\operatorname{Ln}(\mathrm{III})$ for 6-fold, 8-fold and 9-fold coordination and ionic radii of $\mathrm{Ln}(\mathrm{III})$ in aqueous solution (data from ref., ${ }^{2,3}$ ). 


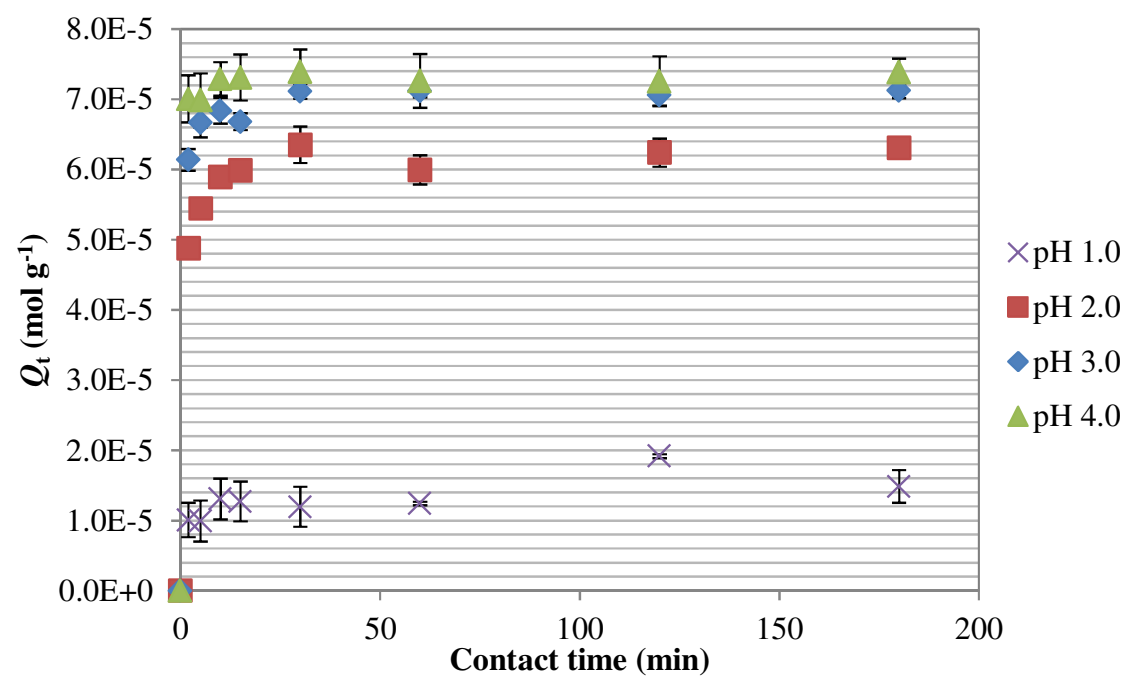

Figure S4. $\mathrm{pH}$ effect on the total adsorbed amount (in $\mathrm{mol} \mathrm{g}^{-1}$ ) of all $\mathrm{Ln}(\mathrm{III})$ on dMNP-DTPA conjugates at different time ( room temperature).

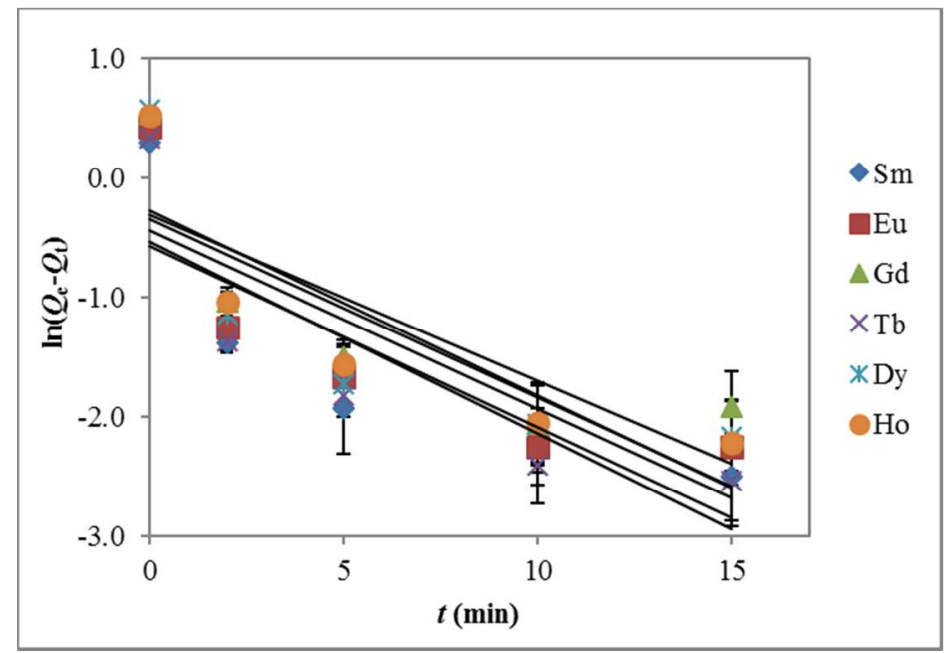

Figure S5. Lagergren plots for sorption of Ln(III) ions on dMNP-DTPA (pH 3.0 acetate buffer, room temperature). 
Table S1. Pseudo-first-order kinetic parameters for Ln(III) sorption on dMNP-DTPA.

\begin{tabular}{|c|c|c|c|c|}
\hline Element & $\boldsymbol{Q}_{\boldsymbol{e}}(\boldsymbol{e x p}).\left(\boldsymbol{m g} \boldsymbol{L}^{-1}\right)$ & $\boldsymbol{Q}_{\boldsymbol{e}}(\boldsymbol{c a l c}).\left(\boldsymbol{m g} \boldsymbol{L}^{-1}\right)$ & $\boldsymbol{k}_{\boldsymbol{l}}\left(\boldsymbol{m i n}^{-1}\right)$ & $\boldsymbol{R}^{2}$ \\
\hline La & 0.091 & NA & NA & NA \\
Ce & 0.234 & NA & NA & NA \\
Pr & 0.502 & NA & NA & NA \\
Nd & 0.748 & NA & NA & NA \\
Sm & 1.346 & 0.564 & 0.152 & 0.6966 \\
Eu & 1.533 & 0.645 & 0.150 & 0.6867 \\
Gd & 1.721 & 0.737 & 0.139 & 0.6544 \\
Tb & 1.402 & 0.589 & 0.161 & 0.7195 \\
Dy & 1.787 & 0.708 & 0.149 & 0.6580 \\
Ho & 1.692 & 0.759 & 0.155 & 0.7327 \\
\hline
\end{tabular}

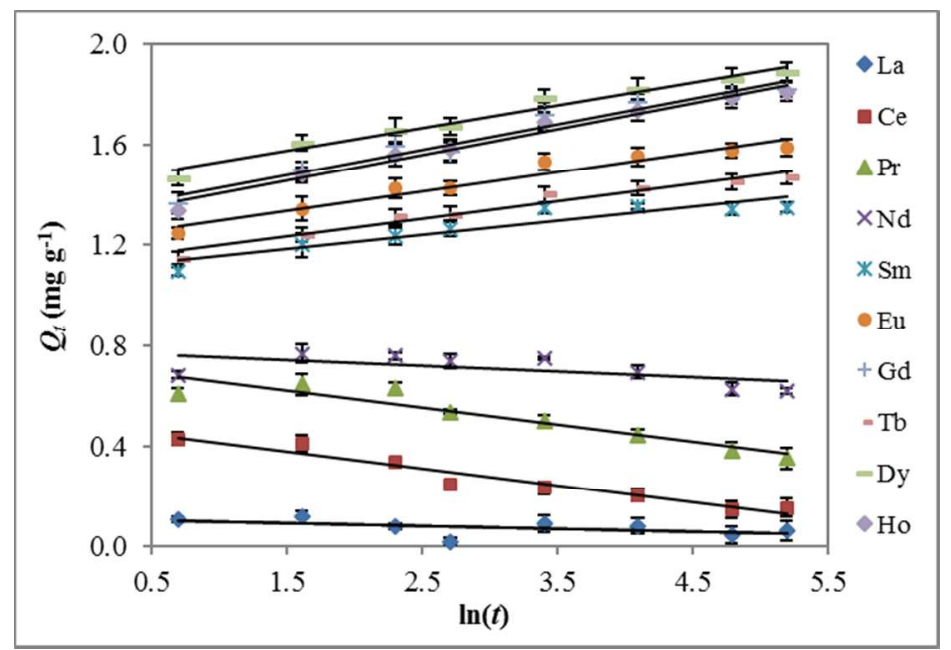

Figure S6. Elovich plots for sorption of $\mathrm{Ln}(\mathrm{III})$ ions on dMNP-DTPA (pH 3.0 acetate buffer, room temperature). 
Table S2. Elovich kinetic parameters for Ln(III) sorption on dMNP-DTPA.

\begin{tabular}{|c|c|c|c|c|c|}
\hline Element & $Q_{e}(\exp ).\left(m g L^{-1}\right)$ & $Q_{e}($ calc. $)\left(m g L^{-1}\right)$ & $\alpha\left(g \mathrm{mg}^{-1} \mathrm{~min}^{-2}\right)$ & $\beta\left(m g g^{-1} \min ^{-1}\right)$ & $R^{2}$ \\
\hline La & 0.091 & 0.072 & $-3.90 \mathrm{E}-03$ & -0.011 & 0.2718 \\
\hline $\mathrm{Ce}$ & 0.234 & 0.250 & $-1.25 \mathrm{E}-02$ & -0.068 & 0.9361 \\
\hline Pr & 0.502 & 0.492 & $-3.18 \mathrm{E}-04$ & -0.067 & 0.8787 \\
\hline Nd & 0.748 & 0.696 & $-1.16 \mathrm{E}-13$ & -0.023 & 0.395 \\
\hline Sm & 1.346 & 1.291 & $6.63 \mathrm{E}+09$ & 0.056 & 0.8665 \\
\hline Eu & 1.533 & 1.486 & $1.09 \mathrm{E}+08$ & 0.077 & 0.9486 \\
\hline Gd & 1.721 & 1.673 & $4.97 \mathrm{E}+06$ & 0.101 & 0.9607 \\
\hline $\mathbf{T b}$ & 1.402 & 1.368 & $1.34 \mathrm{E}+08$ & 0.070 & 0.9622 \\
\hline Dy & 1.787 & 1.748 & $9.60 \mathrm{E}+07$ & 0.090 & 0.9691 \\
\hline Ho & 1.692 & 1.655 & $2.96 \mathrm{E}+06$ & 0.103 & 0.9749 \\
\hline
\end{tabular}

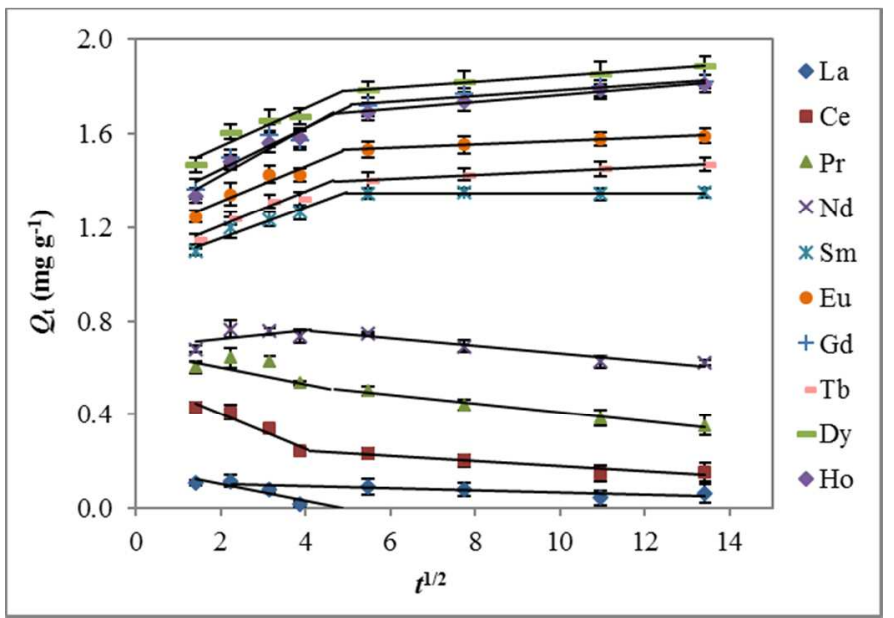

Figure S7. Intra-particle diffusion plots for sorption of $\mathrm{Ln}(\mathrm{III})$ ions on dMNP-DTPA (pH 3.0 acetate buffer, room temperature). 
Table S3. Intra-particle diffusion kinetic parameters for Ln(III) sorption on dMNP-DTPA.

\begin{tabular}{|c|c|c|c|c|c|c|}
\hline Time Range & \multicolumn{3}{|c|}{$\mathbf{0 - 1 5}$ min } & \multicolumn{3}{c|}{$\mathbf{3 0 - 1 8 0 ~ m i n}$} \\
\hline Element & $\boldsymbol{k}_{\boldsymbol{i d}}$ & $\boldsymbol{C}$ & $\boldsymbol{R}^{\mathbf{2}}$ & $\boldsymbol{k}_{\boldsymbol{i d}}$ & $\boldsymbol{C}$ & $\boldsymbol{R}^{\mathbf{2}}$ \\
\hline $\mathbf{L a}$ & -0.036 & 0.177 & 0.7736 & -0.005 & 0.113 & 0.5929 \\
$\mathbf{C e}$ & -0.075 & 0.556 & 0.9183 & -0.011 & 0.289 & 0.8820 \\
$\mathbf{P r}$ & -0.026 & 0.671 & 0.3221 & -0.019 & 0.595 & 0.9810 \\
$\mathbf{N d}$ & 0.019 & 0.684 & 0.2871 & -0.017 & 0.828 & 0.9296 \\
$\mathbf{S m}$ & 0.066 & 1.023 & 0.9061 & 0.000 & 1.349 & 0.0011 \\
$\mathbf{E u}$ & 0.077 & 1.157 & 0.9073 & 0.007 & 1.496 & 0.9840 \\
$\mathbf{G d}$ & 0.088 & 1.272 & 0.8409 & 0.012 & 1.663 & 0.9615 \\
Tb & 0.072 & 1.064 & 0.9296 & 0.008 & 1.359 & 0.9929 \\
$\mathbf{D y}$ & 0.080 & 1.387 & 0.8537 & 0.012 & 1.722 & 0.9957 \\
$\mathbf{H o}$ & 0.100 & 1.224 & 0.9107 & 0.015 & 1.615 & 0.9859 \\
\hline
\end{tabular}

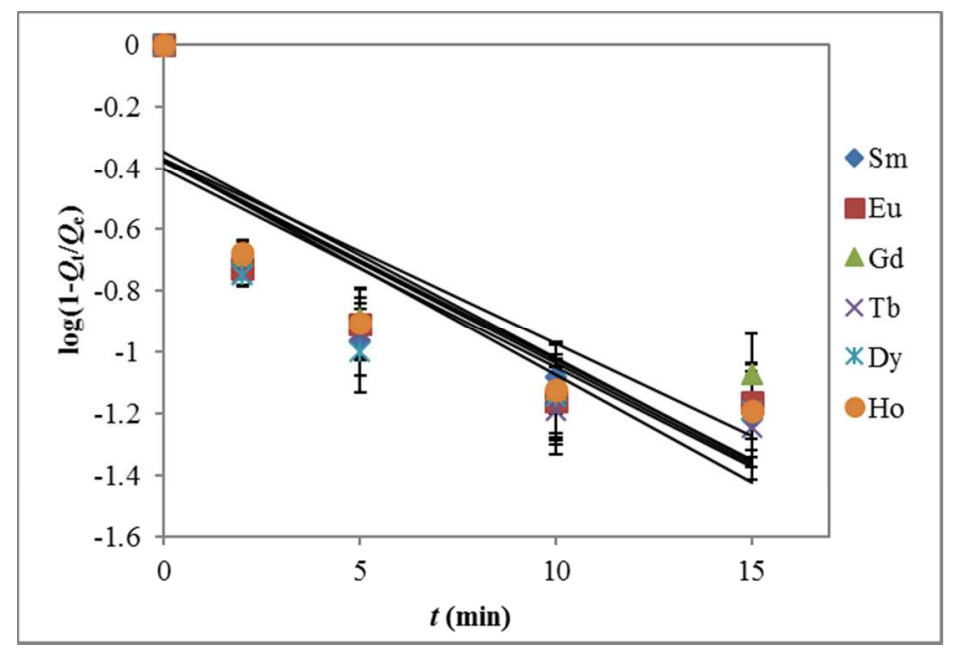

Figure S8. Liquid film diffusion plots for sorption of $\mathrm{Ln}(\mathrm{III})$ ions on dMNP-DTPA (pH 3.0 acetate buffer, room temperature). 
Table S4. Liquid film diffusion kinetic parameters for $\operatorname{Ln}(\mathrm{III})$ sorption on dMNP-DTPA.

\begin{tabular}{|c|c|c|c|c|}
\hline Element & $\boldsymbol{Q e}(\boldsymbol{e x p})\left(\boldsymbol{m g} \boldsymbol{L}^{-1}\right)$ & $\boldsymbol{Q e}(\boldsymbol{c a l c}).\left(\boldsymbol{m g} \boldsymbol{L}^{-1}\right)$ & $\boldsymbol{K}_{\boldsymbol{f d}}\left(\mathbf{m i n}^{-1}\right)$ & $\boldsymbol{R}^{2}$ \\
\hline La & 0.091 & NA & NA & NA \\
Ce & 0.234 & NA & NA & NA \\
Pr & 0.502 & NA & NA & NA \\
Nd & 0.748 & NA & NA & NA \\
Sm & 1.346 & 1.332 & 0.152 & 0.6966 \\
Eu & 1.533 & 1.516 & 0.150 & 0.6867 \\
Gd & 1.721 & 1.695 & 0.139 & 0.6544 \\
Tb & 1.402 & 1.391 & 0.161 & 0.7195 \\
Dy & 1.787 & 1.767 & 0.149 & 0.6580 \\
Ho & 1.692 & 1.676 & 0.155 & 0.7327 \\
\hline
\end{tabular}

\section{References:}

(1) Turanov, A. N.; Karandashev, V. K.; Baulin, V. E. Extraction of Metal Chloride Complexes by Phosphoryl-Containing Podands. Solvent Extr. Ion Exch. 1996, 14 (2), 227-245.

(2) Shannon, R. D. Revised Effective Ionic Radii and Systematic Studies of Interatomic Distances in Halides and Chalcogenides. Acta Crystallogr., Sect. A 1976, 32 (5), 751-767.

(3) D’Angelo, P.; Zitolo, A.; Migliorati, V.; Chillemi, G.; Duvail, M.; Vitorge, P.; Abadie, S.; Spezia, R. Revised Ionic Radii of Lanthanoid(III) Ions in Aqueous Solution. Inorg. Chem. 2011, 50 (10), 4572-4579. 